\title{
CONNECTING VOLUME EFFECTS ON DYNAMICS OF PNEUMATIC PRESSURE MEASUREMENT SYSTEMS
}

\author{
J. Kutin ${ }^{1}$, A. Svete ${ }^{2}$ \\ ${ }^{1}$ University of Ljubljana, Faculty of Mechanical Engineering, Ljubljana, Slovenia, joze.kutin@ @s.uni-lj \\ ${ }^{2}$ University of Ljubljana, Faculty of Mechanical Engineering, Ljubljana, Slovenia, andrej.svete@ fs.uni-lj
}

\begin{abstract}
:
The pressure sensors are often placed at a certain distance from the measured object. Beside the properties of the transmission fluid, dynamic characteristics of such a measurement system depends on the geometry and dimensions of the connecting elements. So far made research works have shown that the internal volume of the pressure sensor can have a large influence on the dynamic response. This paper is focused on theoretical analysis of the effects of the sensor volume on characteristic parameters of both the frequency and the time response of the system under discussion with a gas medium.
\end{abstract}

Keywords: pressure measurement; pressure transmission; dynamic response; sensor volume; connecting tube

\section{INTRODUCTION}

The dynamic response of the pressure measurement system depends on dynamic characteristics of its constructional components. Because the pressure sensors are often placed at a certain distance from the measured object, an additional pressure transmission system is formed in-between. The dynamic response of the pressure transmission depends on geometry, dimensions and stiffness of the connecting elements, as well as thermodynamic and transport properties of the transmission fluid.

The most frequently studied physical model of the pressure transmission system consists from a straight cylindrical connecting tube and an internal volume of the sensor (Figure 1). Following the work of Bergh and Tijdeman [1] the authors of this paper derived one of the latest, most sophisticated versions of the linear mathematical model, which is applicable for both pneumatic and hydraulic pressure measurement systems, and considers viscous, thermal and compliance effects [2]. This improved mathematical model was also experimentally validated for the case of pneumatic pressure measurement systems [3].

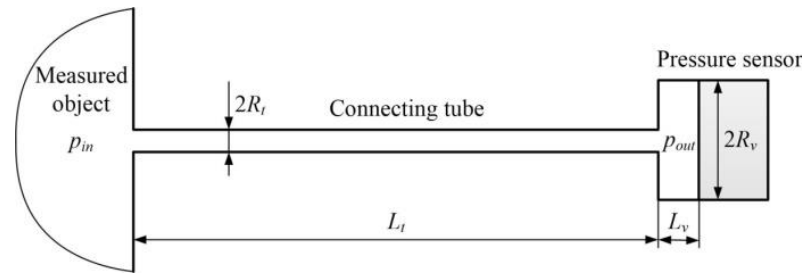

Figure 1: Physical model of the pressure transmission system

In some limited frequency ranges the dynamics of the pressure transmission system can be well described by low-order lumped-parameter mathematical models; e.g., the second-order model with two parameters (natural frequency, damping ratio) or the first-order model with one parameter (time constant) [4-7]. In [2] the authors derived analytical approximations of these lumped parameters by applying an asymptotic series expansion to the complete mathematical model.

The paper is organized as follows. Section 2 presents the mathematical model for the frequency and the step response of the pressure transmission system and its analytical approximations. Section 3 discusses effects of dimensions on characteristic parameters of dynamic response and exposes potential directions for optimal design of the pressure transmission systems. Section 4 shortly summarizes conclusions about the effects of the sensor volume.

\section{MATHEMATICAL MODEL}

The presented mathematical model deals with the pressure transmission system that consists of the cylindrical connecting tube with radius $R_{t}$ and length $L_{t}$ and the cylindrical sensor volume with radius $R_{v}$ and length $L_{v}$ (Figure 1). It is based on the following main assumptions: the internal radius of the tube is small enough in comparison with its length to neglect the end-flow effects, the dimensions of the sensor volume are small enough to neglect fluid-wave effects, and all the pressure variations in the system have small enough amplitudes for validity of the linear model. 


\subsection{Complete model}

The complex frequency response function of the pressure measurement system under discussion can be written as:

$$
G(\mathrm{i} \omega)=\frac{P_{\text {out }}}{P_{\text {in }}}=\frac{1}{\cosh \Gamma+\frac{V_{v}}{V_{t}} \frac{n_{t}}{n_{v}} \Gamma \sinh \Gamma},
$$

where $V_{t}=\pi R_{t}^{2} L_{t}$ is the internal volume of the tube, $V_{v}=\pi R_{v}^{2} L_{v}$ is the internal volume of the sensor, $\Gamma$ is the propagation wavenumber, and $n_{t}$ and $n_{v}$ are the effective thermodynamic indexes for the gas in the tube and the sensor volume, respectively (see [2] for the complete expressions for $\Gamma, n_{t}$ and $n_{v}$, including the wall compliance effects).

The time step response is calculated from the frequency response function by using the Fourier transform, considering the rectangular pulse of the input pressure signal. The Fourier transform of the rectangular pulse with the length $T_{p u l s e}$ is defined as:

$P_{i n}(\mathrm{i} \omega)=\int_{-\infty}^{\infty} p_{i n}(t) \mathrm{e}^{-\mathrm{i} \omega t} \mathrm{~d} t=\frac{1-\mathrm{e}^{-\mathrm{i} \omega T_{\text {puse }}}}{\mathrm{i} \omega}$.

The output signal in the frequency domain is determined as:

$$
P_{\text {out }}(\mathrm{i} \omega)=G(\mathrm{i} \omega) P_{\text {in }}(\mathrm{i} \omega) \text {, }
$$

and the time response is calculated by its inverse Fourier transform:

$$
p_{\text {out }}(t)=\frac{1}{2 \pi} \int_{-\infty}^{\infty} P_{\text {out }}(\mathrm{i} \omega) \mathrm{e}^{\mathrm{i} \omega t} \mathrm{~d} \omega .
$$

With the intention to improve the efficiency of calculations, the procedure presented by equations (2-4) is implemented in calculations in this paper by using the discrete Fourier transform. The corresponding sampling frequency and the number of samples were adapted in a way to assure that errors in calculations of all presented response times are lower than $10^{-3}$.

\subsection{Analytical approximations}

The study in this paper is limited to the pressure transmission systems with the gas medium, where the wall compliance effects are usually not of much importance, so the presented analytical approximations taken from ref. [2] do not consider the compliance effects.

For the connecting tubes with relatively small internal diameters (overdamped conditions) the pressure transmission system can be approximated with the first-order low-wavenumber model:

$$
G_{1}(\mathrm{i} \omega)=\frac{1}{1+\tau \mathrm{i} \omega},
$$

which is defined with one parameter, i.e., the time constant $\tau$ :

$$
\tau=\frac{8 \mu L_{t}^{2}}{\rho c^{2} R_{t}^{2}}\left(\frac{1}{2}+\frac{V_{v}}{V_{t}}\right),
$$

where $c, \rho$ and $\mu$ are the speed of sound, density and dynamic viscosity of gas, respectively. The amplitude frequency response of the first order system is defined as:

$\left|G_{1}(\mathrm{i} \omega)\right|=\frac{1}{\sqrt{1+(\tau \omega)^{2}}}$,

and the step response can be defined as:

$p_{\text {out }, 1}(t)=1-\mathrm{e}^{-t / \tau}$.

For the connecting tubes with relatively large internal diameters (underdamped conditions) the pressure transmission system can be approximated with the second-order high-wavenumber model:

$$
G_{2}(\mathrm{i} \omega)=\frac{1}{1+2 \zeta \mathrm{i} \omega / \omega_{n}-\omega^{2} / \omega_{n}{ }^{2}},
$$

which is defined with two parameters, i.e., the natural frequency $\omega_{n}$ and the damping ratio $\zeta$ :

$$
\begin{aligned}
& \omega_{n}=\frac{c}{L_{t}} \sqrt{\frac{1}{\frac{1}{2}+\frac{V_{v}}{V_{t}}}} \\
& \zeta=\frac{\sqrt{2}}{2 R_{t}} \sqrt{\frac{\mu}{\rho \omega_{n}}}\left(\frac{\omega_{n} L_{t}}{c}\right)^{2}\left(\frac{1+\delta_{t}}{2}+\frac{V_{v}}{V_{t}}\left(1+\delta_{v}\right)\right),
\end{aligned}
$$

where $\delta_{t}$ and $\delta_{v}$ represent the thermal effects for the gas in the tube and the sensor volume, respectively:

$$
\delta_{t}=\frac{\gamma-1}{\sqrt{\operatorname{Pr}}}, \delta_{v}=\frac{R_{t}}{L_{v}}\left(1+\frac{L_{v}}{R_{v}}\right) \frac{\gamma-1}{\sqrt{\mathrm{Pr}}},
$$

where $\gamma$ is the heat capacity ratio and $\operatorname{Pr}$ is the Prandtl number. For $\zeta<<1$ the amplitude frequency response of the second order system can be approximated as:

$\left|G_{2}(\mathrm{i} \omega)\right| \approx \frac{1}{1-\omega^{2} / \omega_{n}^{2}}$,

and the lower envelope of the oscillating step response can be approximated as:

$p_{\text {out }, 2}(t) \approx 1-\mathrm{e}^{-\omega_{n} \zeta t}$. 


\section{RESULTS}

\subsection{Frequency bandwidth}

Characteristics of the frequency response are analysed in terms of the frequency bandwidth, which is defined as the range of frequencies over which the relative amplitude dynamic errors do not exceed $10^{-2}$ or $1 \%$. The bandwidth limit frequency $f_{\text {lim }}$ is calculated as the minimum frequency, where the amplitude ratio reaches 0.99 or 1.01 . Figure 2 shows variations of the limit frequency for different dimensions of the pressure transmission system with dry air $\left(\rho=1.19 \mathrm{~kg} / \mathrm{m}^{3}, c=343 \mathrm{~m} / \mathrm{s}, \mu=18.2\right.$ $\mu \mathrm{Pa} \mathrm{s}, \gamma=1.4, \operatorname{Pr}=0.71)$.

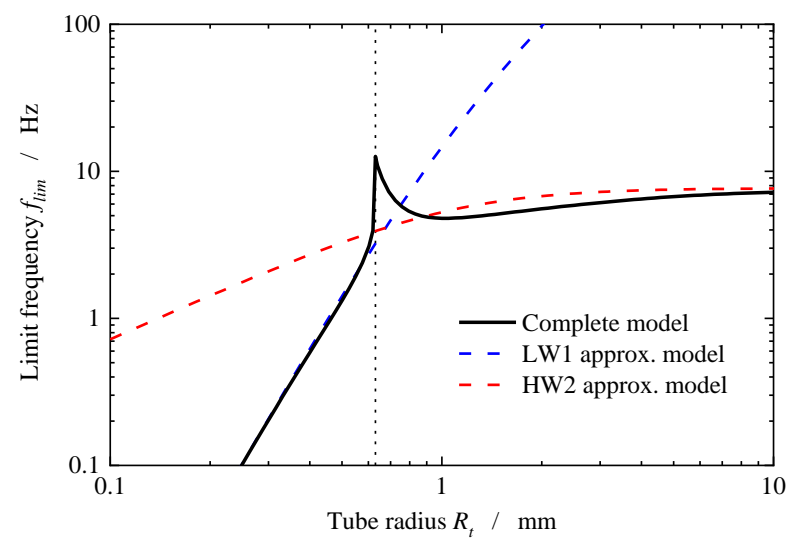

(a) Sensor volume $V_{v}=1.77 \mathrm{~cm}^{3}\left(L_{v}=2.5 \mathrm{~mm}, R_{v}=15 \mathrm{~mm}\right)$

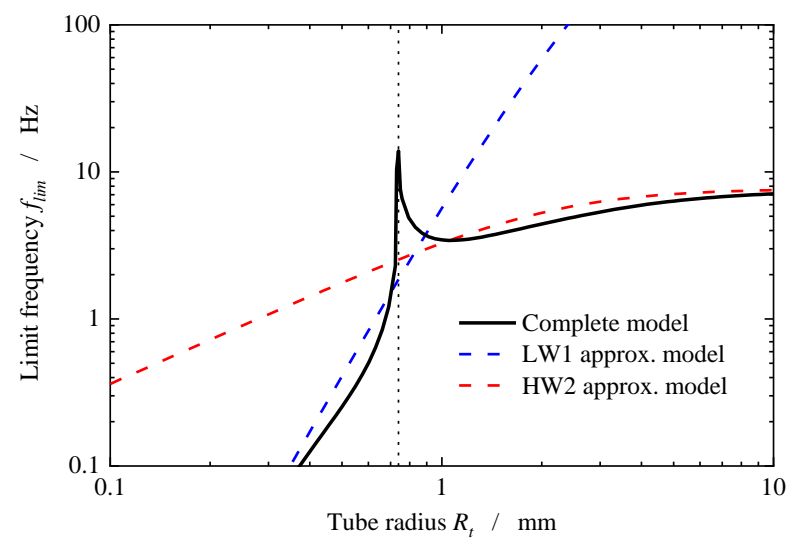

(b) Sensor volume $V_{v}=7.07 \mathrm{~cm}^{3}\left(L_{v}=10 \mathrm{~mm}, R_{v}=15 \mathrm{~mm}\right)$

Figure 2: Variations of the limit frequency with the tube radius for two different sensor volumes (tube length $L_{t}=$ $1 \mathrm{~m}$, dry air)

The dashed lines in Figure 2 represent the results of the first-order low-wavenumber (LW1) and second-order high-wavenumber (HW2) approximation models. The LW1 limit frequency is determined using equation (7):

$\frac{1}{\sqrt{1+\left(\tau \omega_{l i m}^{(L)}\right)^{2}}}=1-e_{r}$,

which results in: $f_{\text {lim }}^{(L)}=\frac{\omega_{\text {lim }}^{(L)}}{2 \pi}=\frac{1}{2 \pi \tau} \sqrt{\frac{1}{\left(1-e_{r}\right)^{2}}-1}$.

The HW2 limit frequency is determined using equation (13):

$\frac{1}{1-\omega_{\text {lim }}^{(H) 2} / \omega_{n}{ }^{2}}=1+e_{r}$,

which results in:

$f_{\text {lim }}^{(H)}=\frac{\omega_{l i m}^{(H)}}{2 \pi}=\frac{\omega_{n}}{2 \pi} \sqrt{1-\frac{1}{1+e_{r}}}$

The LW1 model predicts the limit frequency relatively well for thiner connecting tubes. The frequency bandwidth is inversely proportional to the time constant $\tau$ and, therefore, is inversely proportional to $\left(1 / 2+V_{v} / V_{t}\right)$. The HW2 model predicts the limit frequency relatively well for thicker connecting tubes. The frequency bandwidth is proportional to $\omega_{n}$ and, therefore, is inversely proportional to $\left(1 / 2+V_{v} / V_{t}\right)^{1 / 2}$.

The frequency bandwidth is the largest in the intermediate region of validity of the lowwavenumber and high-wavenumber approximate solutions. For the presented simulation results the largest limit frequency is found at the tube radius of $0.63 \mathrm{~mm}$ for the smaller sensor volume (Figure 2(a)) and at the tube radius of $0.74 \mathrm{~mm}$ for the larger sensor volume (Figure 2(b)). The optimal tube radius that results in the largest frequency bandwidth can be well predicted by the intersection of both approximate models.

\subsection{Settling time}

Characteristics of the step transient response are analysed in terms of the settling time, which is defined as the minimum time that the normalized response needs to fully settle within 0.99 and 1.01; i.e., the relative dynamic errors of the response do not exceed $10^{-2}$ or $1 \%$. Figure 3 shows variations of the settling time for different dimensions of the pressure transmission system.

The dashed lines in Figure 3 represent the results of the first-order low-wavenumber (LW1) and second-order high-wavenumber approximation models. The LW1 settling time is determined using equation (8):

$1-\mathrm{e}^{-\frac{t_{s t}^{(L)}}{\tau}}=1-e_{r}$,

which results in:

$t_{\text {set }}^{(L)}=-\tau \ln e_{r}$. 


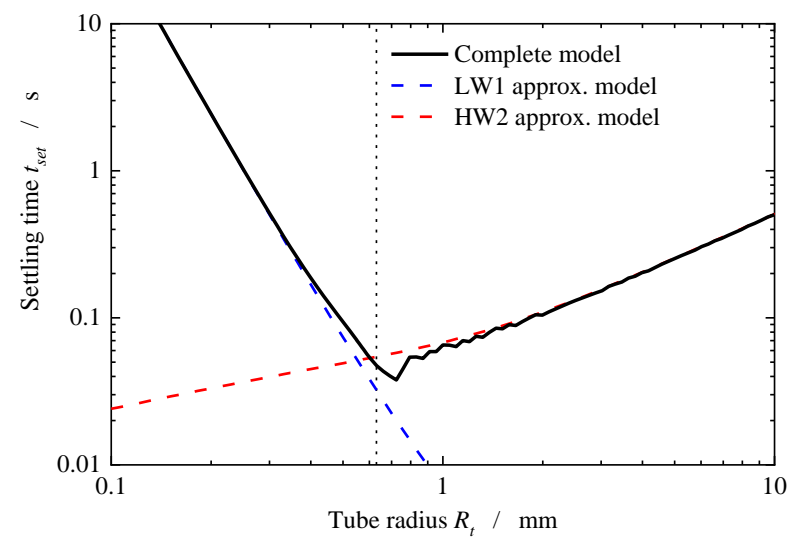

(a) Sensor volume $V_{v}=1.77 \mathrm{~cm}^{3}\left(L_{v}=2.5 \mathrm{~mm}, R_{v}=15 \mathrm{~mm}\right)$

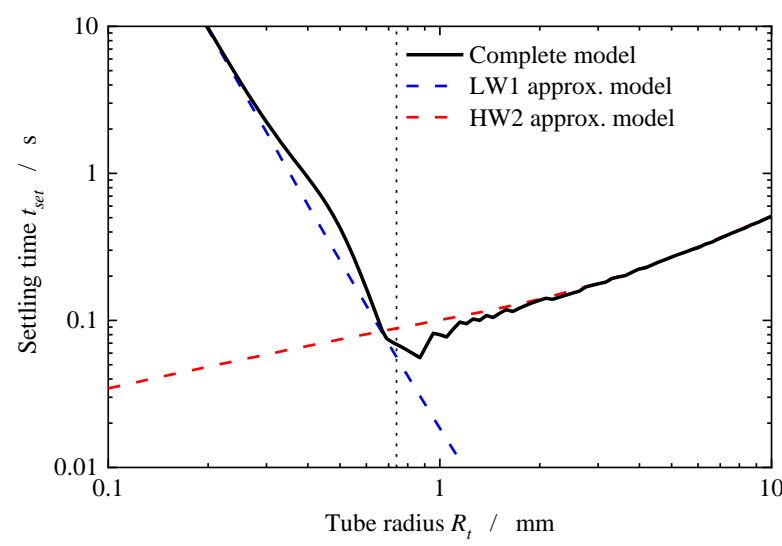

(b) Sensor volume $V_{v}=7.07 \mathrm{~cm}^{3}\left(L_{v}=10 \mathrm{~mm}, R_{v}=15 \mathrm{~mm}\right)$

Figure 3: Variations of the settling time with the tube radius for two different sensor volumes (tube length $L_{t}=$ $1 \mathrm{~m}$, dry air)

The HW2 settling time is determined using equation (14):

$1-\mathrm{e}^{-\omega_{n} t_{s e t}^{(H)}}=1-e_{r}$,

which results in:

$t_{\text {set }}^{(H)}=-\frac{1}{\omega_{n} \zeta} \ln e_{r}$.

The LW1 model predicts the settling time relatively well for thiner connecting tubes. The settling time is proportional to the time constant $\tau$ and, therefore, is proportional to $\left(1 / 2+V_{v} / V_{t}\right)$. The HW2 model predicts the settling time relatively well for thicker connecting tubes. The settling time is inversely proportional to $\omega_{n} \zeta$ and, therefore, is proportional to:

$t_{s e t}^{(H)} \propto \frac{1}{\omega_{n} \zeta} \propto \frac{\left(\frac{1}{2}+\frac{V_{v}}{V_{t}}\right)^{5 / 4}}{\frac{1}{2}\left(1+\delta_{t}\right)+\frac{V_{v}}{V_{t}}\left(1+\delta_{v}\right)} ;$ so if the thermal effects are neglected $\left(\delta_{v} \rightarrow 0, \delta_{t} \rightarrow\right.$ $0)$, the settling time is found proportional to $(1 / 2+$ $\left.V_{v} / V_{t}\right)^{1 / 4}$.

Similar to the findings about the frequency bandwidth, the fastest response is found in the intermediate region of validity of the lowwavenumber and high-wavenumber approximate solutions. The smallest settling time occurs at a bit higher tube radius in comparison with the tube radius that results in the largest limit frequency (marked in Figure 3 with the vertical dot line).

\section{SUMMARY}

As could be expected, the dynamic response of the pressure transmission system is deteriorated with the increased sensor volume. These effects significantly depend on dimensions of the connecting tube. Both the limit frequency of the frequency response and the settling time of the step response are influenced by the sensor volume in terms of the magnitude of $\left(1 / 2+V_{v} / V_{t}\right)$. Therefore, the effects of the sensor volume can be neglected if $V_{v}<<1 / 2 V_{t}$.

\section{REFERENCES}

[1] H. Bergh, H. Tijdeman H, Theoretical and Experimental Results for the Dynamic Response of Pressure Measuring Systems. Amsterdam: Nat. Aero- and Astronautical Res. Inst., Report NLR-TR F.238, 1965.

[2] J. Kutin, A. Svete, "On the Theory of the Frequency Response of Gas and Liquid Pressure Measurement Systems with Connecting Tubes", Measurement Science and Technology, vol. 29, pp. 125108, November 2018.

[3] A. Svete, J. Kutin, "Experimental Validation of an Improved Mathematical Model for Pneumatic Pressure Measurement Systems with Connecting Tubes", Measurement Science and Technology, vol. 31, pp. 015101, October 2019.

[4] E. O. Doebelin, Measurement Systems: Application and Design. Boston, MA: McGrawHill, 2004.

[5] S. A. Whitmore, B. Fox B, "Improved Accuracy, Second-Order Response Model for Pressure Sensing Systems", Journal of Aircraft, vol. 46, pp. 491-500, March-April 2009.

[6] B. F. Hall, T. Povey, "A Practical Model for Pressure Probe System Response Estimation (with Review of Existing Models)", Measurement Science and Technology, vol. 29, pp. 1-12, February 2018.

[7] I. Bajsić, J. Kutin, T. Žagar, "Response Time of a Pressure Measurement System with a Connecting Tube", Instrumentation Science and Technology, vol. 35, pp. 399-409, June 2007. 